\section{Diferenças regionais de conhecimento, opinião e uso de contraceptivo de emergência entre universitários brasileiros de cursos da área de saúde}

\author{
Regional differences in knowledge, attitudes, and \\ practice in emergency contraceptive use among \\ health sciences university students in Brazil
}

\author{
1 Universidade Federal de São \\ Paulo, São Paulo, Brasil. \\ 2 Universidade Federal do \\ Rio Grande do Norte, Natal, \\ Brasil. \\ 3 Universidade Federal de \\ Goiás, Goiânia, Brasil. \\ ${ }^{4}$ Universidade Federal \\ de Santa Catarina, \\ Florianópolis, Brasil. \\ Correspondência \\ F. C. Silva \\ Universidade Federal de São \\ Paulo. \\ Rua Botucatu 715, São Paulo, \\ SP 04023-062, Brasil. \\ flavia_calanca@uol.com.br
}

\begin{abstract}
The aim of this study was to evaluate regional differences in knowledge, attitudes, and practice in emergency contraception use among Brazilian university students. A sample of university students answered a semi-structured questionnaire on knowledge, attitudes, and practice related to emergency contraception and sexual behavior. Fisher's exact test and ANOVA were used for statistical analysis. Statistical significance was set at $p<0.05$. Ninety-six percent $(n=588)$ of the students had heard of emergency contraception, and $19 \%(n=111)$ knew all the situations in which emergency contraception is indicated, with statistical differences between regions of the country. Forty-two percent of sexually active women in the sample had already used emergency contraception; 35\% ( $n=207)$ of students equated emergency contraception with abortion; and $81 \%$ ( $n=$ 473) thought emergency contraception involves health risks. No significant difference was observed between regions of the country regarding use and attitudes towards emergency contraception. Inter-regional differences in knowledge had no impact on students' attitudes and practice in emergency contraception. National awarenessraising campaigns are needed to improve knowledge on emergency contraception.
\end{abstract}

Postcoital Contraception; Adolescent Behavior; Adolescent Medicine; Adolescent; Sexuality
Flávia Calanca da Silva ${ }^{1}$ Maria Sylvia de Souza Vitalle 1 Hélcio de Sousa Maranhão 2 Maria Helena Alves Canuto 3 Maria Marlene de Souza Pires 4 Mauro Fisberg ${ }^{1}$

\section{Introdução}

A adolescência é considerada a melhor fase da vida ${ }^{1}$. É nesse período que os potenciais físicos e psíquicos estão no auge, mas, devido às características próprias do desenvolvimento psicoemocional do adolescente, constitui-se momento de extrema vulnerabilidade a riscos 1 .

A atividade sexual tem início cada vez mais precocemente. Documento publicado pelo Ministério da Saúde em 2008 revelou que na faixa etária de 15-19 anos, entre os anos de 1996 e 2006, houve considerável aumento da proporção de mulheres sexualmente ativas, expondo, mais precocemente e por mais tempo, estas mulheres a eventos reprodutivos (gravidez e abortos) e doenças sexualmente transmissíveis (DST) ${ }^{2}$.

A gravidez na adolescência é tema polêmico e controverso nos debates sobre saúde sexual do adolescente; em geral, o discurso "gravidez na adolescência” sempre adquire um tom alarmista e moralista, associando-a à pobreza; desestruturação familiar; riscos sociais, médicos e psicológicos para os sujeitos envolvidos 3,4. Mudanças culturais fizeram com que a mulher desempenhasse novo papel na sociedade, modificando as perspectivas e as possibilidades educacionais e profissionais 3 . Devido a isso, a experiência de engravidar na juventude pode ser considerada elemento perturbador do desenvolvimento ideal desta etapa da vida, agra- 
vando ou determinando situação de marginalidade econômica ou vulnerabilidade social 3 .

Embora as taxas de gestação em mulheres menores de 20 anos de idade venham apresentando tendência a declínio em todo o mundo, elas ainda são muito elevadas 5. No Brasil, em 2006, 20\% dos nascimentos ocorridos foram provenientes de mães menores de 20 anos, sendo que a distribuição geográfica evidencia maiores freqüências nas regiões Norte e Nordeste (29\% e $25,5 \%$, respectivamente) e menores no Sudeste e Sul, respectivamente, $18 \%$ e $19,5 \% 6$.

Em relação à primeira gravidez, estudos indicam que entre $60 \%$ e $83,7 \%$ das adolescentes não pretendiam engravidar, mostrando que a maternidade precoce geralmente não é planejada e/ ou desejada 7,8,9,10. Apesar dos riscos à saúde e da legalidade restrita do aborto no Brasil, muitas adolescentes pensam e efetivamente tentam interromper a gestação 7,9. A maioria das pesquisas sobre aborto no Brasil está concentrada em hospitais públicos, com mulheres admitidas para tratamento do aborto incompleto, restringindose, portanto, aos abortos que apresentaram complicações, subestimando, desta forma, a sua real magnitude 11. Apesar das dificuldades, estima-se uma incidência aproximada de 30 abortos para cada 100 nascidos vivos, sendo que entre $7 \%$ e 9\% do total destes abortos realizados ocorreram na adolescência 9,12.

Programas objetivando prevenir a gravidez indesejada na adolescência são especialmente importantes. Acesso fácil e não burocrático a serviços de planejamento familiar e métodos contraceptivos são de extrema relevância.

Dentre as várias formas de contracepção existentes, a anticoncepção de emergência é o método que previne gravidez após a relação sexual, podendo ser uma estratégia interessante para diminuir a incidência de gravidez indesejada e as taxas de abortos ilegais entre adolescentes. Embora a "contracepção de emergência" seja assunto polêmico, trata-se de método seguro, tanto do ponto de vista dos riscos à saúde como em relação aos aspectos comportamentais. Apesar da possibilidade do uso indiscriminado ser preocupação constante descrita na literatura, tanto entre usuários como entre profissionais de saúde (os médicos temem prescrever anticoncepção de emergência para adolescentes receando que esta atitude possa promover comportamento sexual irresponsável ou uso inadequado), há inúmeros trabalhos que não confirmam esta evidência: conhecer, ser orientada e mesmo possuir prescrição de anticoncepção de emergência não modifica o comportamento sexual de adolescentes e mulheres adultas (não interfere no uso de outros métodos contraceptivos, não leva à promiscuida- de sexual, não interfere no número de parceiros) 13,14,15,16,17,18. Portanto, é um método efetivo, com indicações reservadas a situações especiais ou de exceção, cujo objetivo é prevenir a gravidez inoportuna ou indesejada após relação sexual que, por alguma razão, foi desprotegida, oferecendo à adolescente uma segunda chance de evitar uma gestação não desejada 19,20,21.

Apesar de estarem liberados há mais de 30 anos em muitos países, e no Brasil estarem incluídos nas normas técnicas de planejamento familiar do Ministério da Saúde desde 1996, constituem-se método pouco utilizado 22,23,24. Estudos que avaliam o conhecimento de adolescentes e mulheres adultas sobre anticoncepção de emergência mostram que apesar do método ser conhecido, informações mais específicas, como até quando pode ser utilizado, qual a eficácia do método, o mecanismo de ação e os efeitos colaterais, e onde adquiri-lo, são escassas, contribuindo para uma visão negativa e conseqüente subutilização $23,25,26,27,28,29$. Os trabalhos nacionais que abordam o conhecimento e uso de anticoncepção de emergência entre universitários e estudantes de ensino médio falham em avaliar se os adolescentes realmente conhecem o método; em um destes estudos, apesar da maioria relatar que conhece a anticoncepção de emergência, constatou-se que houve predomínio do uso incorreto, evidenciando conhecimento escasso 30,31,32. Inexistem estudos que mostrem como difere o conhecimento sobre anticoncepção de emergência nas diferentes regiões do Brasil, dificultando a promoção de medidas de saúde dirigidas.

Diante do exposto, objetiva-se com este trabalho avaliar as diferenças inter-regionais existentes em relação ao comportamento sexual, uso de métodos contraceptivos, conhecimento, experiência e opinião de adolescentes universitários sobre contracepção de emergência, bem como a relação entre estas variáveis.

\section{Métodos}

Trata-se de estudo observacional e de corte transversal. Estudantes do primeiro ano de cursos da área da saúde (medicina, enfermagem, nutrição e educação física) de quatro universidades federais do Brasil (Universidade Federal de São Paulo - UNIFESP; Universidade Federal de Santa Catarina - UFSC; Universidade Federal de Goiás - UFG; e Universidade Federal do Rio Grande do Norte - UFRN), representando quatro regiões geográficas do país, foram recrutados no início dos anos letivos de 2006 e 2007 para responder a questionário semi-estruturado e autopreenchível. O objetivo desse recrutamento foi evitar o 
acesso dos estudantes a conteúdo programático que pudesse interferir com as respostas das questões. Dificuldades operacionais (greves) impossibilitaram a coleta de dados na Região Norte do país.

É importante salientar que a escolha dessas universidades foi por conveniência. Como a instituição base do estudo foi a UNIFESP, utilizaram-se universidades federais que possuíam os mesmos cursos da área de saúde, carga horária e número de alunos equivalentes. Foram escolhidos estudantes de universidades federais por representarem de forma teórica a elite do conhecimento entre adolescentes ingressantes em curso superior, baseada na proporção número de candidatos-vagas. Levando-se em consideração que no Brasil cerca de $36 \%$ dos jovens brasileiros cursam o ensino superior e, destes, apenas $24,7 \%$ freqüentam as universidades públicas, tem-se quão disputado e difícil é ingressar neste tipo de instituição 33. De modo geral, os jovens que ingressam em universidades públicas possuem melhor nível de conhecimento, são mais bem preparados e têm mais acesso à informação quando comparados aos que freqüentam instituições privadas ou aos que não ingressaram no Ensino Superior.

Cada questionário continha 10 questões abertas e 31 de múltipla escolha para caracterizar o estudante segundo idade, sexo, curso e universidade freqüentada, nível socioeconômico, conhecimento, opinião e experiência com anticoncepção de emergência; comportamento sexual (uso de métodos contraceptivos, número de parceiros e idade do primeiro intercurso sexual). As questões formuladas para avaliar o conhecimento sobre anticoncepção de emergência abordavam se o adolescente já "tinha ouvido falar” do método, o tempo máximo após o intercurso desprotegido para que a anticoncepção de emergência pudesse ser usada, as indicações, a efetividade, se o método é abortivo e traz riscos à saúde; e a fonte de aquisição destes conhecimentos. Quanto à opinião, eles foram questionados se a possibilidade de usar anticoncepção de emergência estimularia os adolescentes a terem relação sexual desprotegida. Por fim, os estudantes foram indagados se já usaram, usariam ou indicariam o uso da anticoncepção de emergência para a parceira. Aos que usaram, questionou-se como o método foi adquirido, os motivos de uso e quantas vezes a anticoncepção de emergência foi utilizada.

O questionário foi desenvolvido para este fim, com base em estudos similares e foi prétestado em estudantes de universidade particular da cidade de São Paulo para corrigir as imperfeições 13,34,35,36.
Utilizaram-se como critérios de inclusão ter idade menor do que 20 anos (uma vez que o objetivo foi avaliar apenas adolescentes), estar regularmente matriculado no curso escolhido, estar presente em sala de aula no momento da aplicação do questionário, ser procedente da região do país da universidade freqüentada, bem como ter aceitado participar do estudo mediante leitura e assinatura do termo de consentimento livre e esclarecido 37

Por se tratar de menor de idade e por ser a atividade sexual um tema de foro íntimo e pessoal do adolescente, independentemente da autorização dos responsáveis, o termo de consentimento não foi aplicado aos pais dos estudantes para preservação do sigilo. Por serem todos universitários, foram considerados como menores responsáveis.

Para a definição do nível socioeconômico foi utilizado o modelo criado pela Associação Brasileira de Empresas de Pesquisa (ABEP) em 2003 38. Para a análise estatística os estudantes foram classificados de acordo com a região da universidade freqüentada, em sexo masculino e feminino, e agrupados em classes $\mathrm{A}(\mathrm{A} 1+\mathrm{A} 2)$, B $(\mathrm{B} 1+\mathrm{B} 2)$ e Outra $(\mathrm{C}+\mathrm{D}+\mathrm{E})$.

Para as variáveis categóricas usou-se o teste exato de Fisher e para as variáveis contínuas o modelo de Análise de Variância - ANOVA 39,40. A fim de complementar a análise das variáveis categóricas que apresentaram significância estatística foram calculadas as razões de chance. As diferenças foram consideradas estatisticamente significantes quando o valor de $\mathrm{p}$ foi menor do que 0,05 (5\%). Todos os resultados foram gerados utilizando-se o programa SAS versão 8.2 (SAS Inst., Cary, Estados Unidos).

Este estudo está de acordo com a Resolução $n^{o}$. 196/96 do Conselho Nacional de Saúde do Ministério da Saúde, que regulamenta as pesquisas envolvendo seres humanos, tendo sido aprovado pelo Comitê de Ética em Pesquisa do Hospital São Paulo, UNIFESP, nº. 0727/06.

\section{Resultados}

\section{Caracterização da amostra}

Dez por cento dos estudantes matriculados nos cursos não estavam presentes em sala de aula no momento da aplicação do questionário ou eram provenientes de regiões distintas da universidade freqüentada, sendo que 611 alunos responderam à pesquisa.

A caracterização da amostra encontra-se descrita na Tabela 1. No estudo, prevaleceram estudantes do sexo feminino em todas as regiões. 
Tabela 1

Características sociodemográficas da amostra de acordo com a Universidade Federal de origem.

\begin{tabular}{|c|c|c|c|c|c|c|c|c|c|c|c|}
\hline \multirow[t]{2}{*}{ Características } & \multicolumn{2}{|c|}{$\mathrm{SC}(\mathrm{N}=138)$} & \multicolumn{2}{|c|}{$\mathrm{SP}(N=161)$} & \multicolumn{2}{|c|}{$\mathrm{GO}(\mathrm{N}=170)$} & \multicolumn{2}{|c|}{$\mathrm{RN}(\mathrm{N}=142)$} & \multicolumn{2}{|c|}{ Total $(\mathrm{N}=611)$} & \multirow{2}{*}{$\begin{array}{l}\text { Valor } \\
\text { de } p\end{array}$} \\
\hline & $\mathrm{n}$ & $\%$ & $\mathbf{n}$ & $\%$ & $\mathbf{n}$ & $\%$ & $\mathbf{n}$ & $\%$ & $\mathbf{n}$ & $\%$ & \\
\hline Sexo & $n=138$ & & $n=161$ & & $n=170$ & & $n=142$ & & $n=611$ & & \\
\hline Feminino & 93 & 67,4 & 106 & 65,8 & 117 & 68,8 & 88 & 62,0 & 404 & 66,1 & 0,4589 \\
\hline Masculino & 45 & 32,6 & 55 & 34,2 & 53 & 31,2 & 54 & 38,0 & 207 & 33,9 & \\
\hline Média da idade (anos) & $\begin{array}{c}18,20 \pm 0,71 \\
(17-19)\end{array}$ & & $\begin{array}{c}18,29 \pm 0,75 \\
(17-19)\end{array}$ & & $\begin{array}{c}18,03 \pm 0,83 \\
(16-19)\end{array}$ & & $\begin{array}{c}18,35 \pm 0.67 \\
(17-19)\end{array}$ & & $\begin{array}{c}18,25 \pm 0,75 \\
(16-19)\end{array}$ & & 0,2378 \\
\hline Nível socioeconômico *,** & $n=138$ & & $\mathrm{n}=160$ & & $\mathrm{n}=163$ & & $\mathrm{n}=126$ & & $\mathrm{n}=587$ & & \\
\hline A & 47 & 34,1 & 77 & 48,1 & 52 & 31,9 & 40 & 31,8 & 216 & 36,8 & 0,0002 \\
\hline B & 73 & 52,9 & 75 & 46,9 & 81 & 49,7 & 58 & 46,0 & 287 & 48,9 & \\
\hline $\mathrm{C} / \mathrm{D} / \mathrm{E}$ & 18 & 13,0 & 8 & 5,0 & 30 & 18,4 & 28 & 22,2 & 84 & 14,3 & \\
\hline
\end{tabular}

* Estatisticamente significante $(p<0,05)$;

** Nem todos os estudantes responderam a essa questão.

GO: Goiás; RN: Rio Grande do Norte; SC: Santa Catarina; SP: São Paulo.

Observou-se diferença estatisticamente significante do nível socioeconômico entre as regiões ( $p=0,0002)$. Exceto em São Paulo, onde prevaleceu o nível socioeconômico A, nas demais regiões predominaram estudantes do nível social econômico B. Nível socioeconômico C/D foi mais prevalente no Rio Grande do Norte. Não havia na amostra alunos do nível socioeconômico E.

A caracterização dos estudantes de acordo com o comportamento sexual e uso de métodos contraceptivos encontra-se na Tabela 2. Cinqüenta e seis por cento $(n=337)$ dos alunos que participaram do estudo já tinham tido relação sexual, sendo $47,1 \%(\mathrm{n}=187)$ meninas e $73,5 \%$ ( $\mathrm{n}=150)$ meninos $(\mathrm{p}<0,0001)$. Observou-se diferença estatisticamente significante em relação a ter tido ou não intercurso sexual entre as regiões $(p=0,0006)$ : a maior prevalência de atividade sexual foi encontrada em Santa Catarina e a menor foi observada no Rio Grande do Norte. As médias de idade do primeiro intercurso sexual bem como do número de parceiros não mostraram diferença estatística entre as regiões, mas apresentaram diferença entre os gêneros: a idade da primeira relação sexual dos estudantes do sexo masculino foi, em média, um ano abaixo da idade da primeira relação dos estudantes do sexo feminino (15,69 anos versus 16,65 anos, respectivamente); e os estudantes do sexo masculino tiveram, em média, 3,26 parceiros contra 1,65 do sexo feminino ( $\mathrm{p}<0,0001)$. Os métodos contraceptivos mais utilizados pelos adolescentes sexualmente ativos em todas as regiões foram preservativo masculino seguido pelos anticoncepcionais orais hormonais. Cerca de $10 \%$ ( $\mathrm{n}=$
34) dos alunos referiram utilizar coito interrompido e $7,5 \%(n=25)$ relataram fazer tabelinha como formas de contracepção. Cerca de $30 \%$ (n = 97) dos estudantes referiram não usar métodos contraceptivos em todas as relações sexuais. Não se observou diferença estatisticamente significativa entre os sexos e entre adolescentes do sexo masculino por região quando se avaliou a freqüência de uso de métodos contraceptivos, mas esta diferença estava presente entre adolescentes do sexo feminino ( $p=0,0444)$ : a chance de uma estudante de São Paulo usar métodos contraceptivos em todas as relações é a mesma de uma estudante de Santa Catarina e maior que das estudantes de Goiás e do Rio Grande do Norte. Menos de $1 \%(n=3)$ dos pesquisados referiu nunca ter usado nenhum método contraceptivo em suas relações sexuais.

\section{Conhecimento sobre anticoncepção de emergência}

Cerca de 96\% ( $\mathrm{n}=588)$ dos estudantes referiram "já ter ouvido falar" sobre anticoncepção de emergência, sendo 96,8\% ( $\mathrm{n}=390)$ meninas e $95,7 \%(n=198)$ meninos. Observou-se diferença estatisticamente significante em relação a essa informação entre estudantes do sexo feminino nas diferentes regiões $(p=0,0091)$ : a chance de uma estudante de Goiás "já ter ouvido falar" sobre anticoncepção de emergência é maior que a chance das estudantes das outras regiões (Tabela 3).

Menos da metade dos estudantes, 40,7\% ( $\mathrm{n}=$ 238), sabiam que a anticoncepção de emergência 
Tabela 2

Comportamento sexual e uso de contraceptivos da amostra de acordo com a Universidade Federal de origem.

\begin{tabular}{|c|c|c|c|c|c|c|c|c|c|c|c|}
\hline \multirow[t]{2}{*}{ Características } & \multicolumn{2}{|c|}{$\mathrm{SC}(\mathrm{N}=138)$} & \multicolumn{2}{|c|}{$\mathrm{SP}(N=161)$} & \multicolumn{2}{|c|}{$\mathrm{GO}(\mathrm{N}=170)$} & \multicolumn{2}{|c|}{$\mathrm{RN}(\mathrm{N}=142)$} & \multicolumn{2}{|c|}{ Total $(\mathrm{N}=611)$} & \multirow[t]{2}{*}{ Valor de $p$} \\
\hline & $\mathbf{n}$ & $\%$ & $\mathrm{n}$ & $\%$ & $\mathbf{n}$ & $\%$ & $\mathbf{n}$ & $\%$ & $\mathbf{n}$ & $\%$ & \\
\hline Relação Sexual *,** & $\mathrm{n}=135$ & & $n=159$ & & $n=167$ & & $n=140$ & & $n=601$ & & \\
\hline Sim & 93 & 68,9 & 95 & 59,7 & 84 & 50,3 & 65 & 46,4 & 337 & 56,1 & 0,0006 \\
\hline Não & 42 & 31,1 & 64 & 40,3 & 83 & 49,7 & 75 & 53,6 & 264 & 43,9 & \\
\hline $\begin{array}{l}\text { Média da idade da } \\
\text { primeira relação sexual } \\
\text { (anos) ** }\end{array}$ & $\begin{array}{c}16,09 \pm 1,66 \\
(9-19)\end{array}$ & & $\begin{array}{c}16,56 \pm 1,10 \\
(13-19)\end{array}$ & & $\begin{array}{c}16,17 \pm 1,57 \\
(13-19)\end{array}$ & & $\begin{array}{c}16,02 \pm 1,62 \\
(13-19)\end{array}$ & & $\begin{array}{c}16,23 \pm 1,50 \\
(9-19)\end{array}$ & & 0,0838 \\
\hline $\begin{array}{l}\text { Média do número de } \\
\text { parceiros sexuais ** }\end{array}$ & $\begin{array}{c}2,12 \pm 1,80 \\
(1-12)\end{array}$ & & $\begin{array}{l}1,87 \pm 1,64 \\
(1-11)\end{array}$ & & $\begin{array}{l}2,55 \pm 3,27 \\
\quad(1-17)\end{array}$ & & $\begin{array}{c}2,33 \pm 2,11 \\
(1-9)\end{array}$ & & $\begin{array}{c}2,18 \pm 2,26 \\
(1-17)\end{array}$ & & 0.2316 \\
\hline $\begin{array}{l}\text { Métodos contraceptivos } \\
\text { utilizados } \star \star, \star \star \star\end{array}$ & $n=93$ & & $n=95$ & & $\mathrm{n}=83$ & & $\mathrm{n}=63$ & & $n=334$ & & \\
\hline Preservativo masculino & 85 & 91,4 & 90 & 94,7 & 78 & 94,0 & 57 & 90,5 & 310 & 92,8 & 0,6911 \\
\hline Contraceptivos orais * & 57 & 61,3 & 56 & 58,9 & 44 & 53,0 & 19 & 30,2 & 176 & 52,7 & 0,0011 \\
\hline Coito interrompido & 7 & 7,5 & 9 & 9,5 & 8 & 9,6 & 10 & 15,9 & 34 & 10,2 & 0,4015 \\
\hline Camisinha feminina & 3 & 3,2 & 2 & 2,1 & 2 & 2,4 & 0 & 0,0 & 7 & 2,1 & 0,9694 \\
\hline Tabelinha & 2 & 2,2 & 12 & 12,6 & 5 & 6,0 & 6 & 9,5 & 25 & 7,5 & 0,0777 \\
\hline Adesivo & 1 & 1,1 & 0 & 0,0 & 0 & 0,0 & 0 & 0,0 & 1 & 0,3 & 0,9997 \\
\hline Contraceptivo injetável & 0 & 0,0 & 2 & 2,1 & 2 & 2,4 & 5 & 7,9 & 9 & 2,7 & 0,5012 \\
\hline DIU & 0 & 0,0 & 0 & 0,0 & 1 & 1,2 & 0 & 0,0 & 1 & 0,3 & 0,9997 \\
\hline Diafragma & 0 & 0,0 & 1 & 1,1 & 0 & 0,0 & 0 & 0,0 & 1 & 0,3 & 0,9997 \\
\hline Implante & 0 & 0,0 & 0 & 0,0 & 0 & 0,0 & 0 & 0,0 & 0 & 0,0 & 0,9456 \\
\hline Anel vaginal & 0 & 0,0 & 0 & 0,0 & 0 & 0,0 & 1 & 1,6 & 1 & 0,3 & 0,9995 \\
\hline
\end{tabular}

* Estatisticamente significante $(p<0,05)$;

** Nem todos os estudantes responderam a essa questão;

*** Mais de uma alternativa possível.

GO: Goiás; RN: Rio Grande do Norte; SC: Santa Catarina; SP: São Paulo.

deveria ser usada até 72 horas do intercurso sexual desprotegido e cerca de $48 \%$ dos estudantes ( $n=280)$ achavam que a mulher tinha de 24 a 48 horas para usá-la. Não se observou diferença estatisticamente significante entre as regiões para essa variável.

Aproximadamente $19 \%(n=111)$ dos estudantes conheciam precisamente todas as situações nas quais a anticoncepção de emergência está indicada (relação sexual sem uso de método contraceptivo, falha conhecida ou presumida do método em uso de rotina, uso inadequado do contraceptivo e abuso sexual). Cerca de $6 \%$ ( $\mathrm{n}=$ 37) dos adolescentes, embora soubessem alguma indicação da anticoncepção de emergência, responderam que poderia ser usada como método de rotina. Diferença estatística significativa para essa variável foi observada tanto entre estudantes do sexo masculino ( $p<0,0001$ ) como do sexo feminino ( $\mathrm{p}=0,0021)$, nas diferentes regiões. Obteve-se que a chance dos estudantes de Santa Catarina, tanto do sexo masculino como do se- xo feminino, conhecerem as situações de uso é maior que a chance dos estudantes das outras regiões.

Quarenta e oito por cento $(n=283)$ dos estudantes sabiam informar quão efetiva é a anticoncepção de emergência quando comparada aos anticoncepcionais orais hormonais se estes forem usados adequadamente. Encontrou-se diferença estatística significante em ambos os sexos, entre as diferentes regiões. A chance dos estudantes de São Paulo, dos sexos feminino e masculino, saberem sobre a efetividade da anticoncepção de emergência é maior que a chance dos estudantes das outras regiões.

Cerca de $33 \%$ dos alunos relataram que tinham aprendido sobre anticoncepção de emergência com médicos. Observou-se que em Goiás a chance de um estudante adquirir conhecimento sobre anticoncepção de emergência por meio da escola é 6,2 vezes maior do que a chance de aquisição de conhecimento por intermédio de amigos; 33,1 vezes maior do que pela família; 
Tabela 3

Distribuição dos estudantes universitários do sexo feminino e do sexo masculino, de acordo com o conhecimento de anticoncepção de emergência (sim, não e não sei) e universidade federal de origem.

\begin{tabular}{|c|c|c|c|c|c|c|c|c|}
\hline \multirow{2}{*}{$\begin{array}{l}\text { Região } \\
\text { Já ouviu falar de } \\
\text { anticoncepção de } \\
\text { emergência? }\end{array}$} & \multicolumn{4}{|c|}{ Feminino [n (\%)] } & \multicolumn{4}{|c|}{ Masculino [n (\%)] } \\
\hline & $\operatorname{Sim}(n=390)$ & Não $(n=13)$ & & Valor de $p$ * & $\operatorname{Sim}(n=198)$ & Não $(n=9)$ & & Valor de $p$ * \\
\hline SC & $92(23,6)$ & $1(7,7)$ & & 0,0091 & $43(21,7)$ & $2(22,2)$ & & 0,9371 \\
\hline $\mathrm{SP}$ & $105(26,9)$ & $1(7,7)$ & & & $53(26,8)$ & $2(22,2)$ & & \\
\hline GO & $114(29,2)$ & $3(23,1)$ & & & $50(25,2)$ & $3(33,4)$ & & \\
\hline $\mathrm{RN}$ & $79(20,3)$ & $8(61,5)$ & & & $52(26,3)$ & $2(22,2)$ & & \\
\hline $\begin{array}{l}\text { Sabe até quanto tempo } \\
\text { após o intercurso } \\
\text { desprotegido a } \\
\text { anticoncepção de } \\
\text { emergência pode ser } \\
\text { usada? }\end{array}$ & $\begin{array}{c}\operatorname{Sim} \\
(n=171)\end{array}$ & $\begin{array}{c}\text { Não } \\
(n=175)\end{array}$ & $\begin{array}{l}\text { Não sei } \\
(n=41)\end{array}$ & Valor de $p$ * & $\begin{array}{c}\text { Sim } \\
(n=67)\end{array}$ & $\begin{array}{c}\text { Não } \\
(n=105)\end{array}$ & $\begin{array}{l}\text { Não sei } \\
(n=26)\end{array}$ & Valor de $p$ * \\
\hline $\mathrm{SC}$ & $33(19,3)$ & $47(26,9)$ & $10(24,4)$ & 0,0519 & $10(14,9)$ & $27(25,7)$ & $6(23,1)$ & 0,0910 \\
\hline $\mathrm{SP}$ & $54(31,6)$ & $43(24,6)$ & $8(19,5)$ & & $24(35,8)$ & $19(18,1)$ & $10(38,4)$ & \\
\hline GO & $57(33,3)$ & $47(26,9)$ & $9(21,9)$ & & $14(20,9)$ & $30(28,6)$ & $6(23,1)$ & \\
\hline $\mathrm{RN}$ & $27(15,8)$ & $38(27,7)$ & $14(24,2)$ & & $19(28,4)$ & $29(27,6)$ & $4(15,4)$ & \\
\hline $\begin{array}{l}\text { Conhece situações nas } \\
\text { quais a anticoncepção de } \\
\text { emergência está indicada? }\end{array}$ & $\begin{array}{c}\operatorname{Sim} \\
(n=317)\end{array}$ & $\begin{array}{c}\text { Não } \\
(n=73)\end{array}$ & & Valor de $p$ * & $\begin{array}{c}\operatorname{Sim} \\
(n=160)\end{array}$ & $\begin{array}{c}\text { Não } \\
(n=38)\end{array}$ & & Valor de $p$ * \\
\hline SC & $85(26,8)$ & $7(9,6)$ & & 0,0021 & $41(25,6)$ & $2(5,3)$ & & \\
\hline $\mathrm{SP}$ & $77(24,3)$ & $28(38,4)$ & & & $38(23,8)$ & $15(39,5)$ & & $<0,0001$ \\
\hline GO & $88(27,8)$ & $26(35,6)$ & & & $33(20,6)$ & $17(44,7)$ & & \\
\hline $\mathrm{RN}$ & $67(21,1)$ & $12(16,4)$ & & & $48(30,0)$ & $4(10,5)$ & & \\
\hline $\begin{array}{l}\text { Sabe quão efetiva é } \\
\text { a anticoncepção de } \\
\text { emergência quando } \\
\text { comparada com as pílulas } \\
\text { comuns? }\end{array}$ & $\begin{array}{c}\text { Sim } \\
(n=192)\end{array}$ & $\begin{array}{c}\text { Não } \\
(n=117)\end{array}$ & $\begin{array}{l}\text { Não sei } \\
(n=80)\end{array}$ & Valor de $p$ * & $\begin{array}{l}\operatorname{Sim} \\
(n=91)\end{array}$ & $\begin{array}{c}\text { Não } \\
(n=53)\end{array}$ & $\begin{array}{l}\text { Não sei } \\
(n=53)\end{array}$ & Valor de $p$ * \\
\hline $\mathrm{SC}$ & $50(26,0)$ & $25(21,4)$ & $16(20,0)$ & 0,0383 & $22(24,2)$ & $7(13,2)$ & $14(26,4)$ & 0,0408 \\
\hline $\mathrm{SP}$ & $59(30,7)$ & $28(23,9)$ & $18(22,6)$ & & $29(31,8)$ & $13(24,5)$ & $11(20,7)$ & \\
\hline $\mathrm{GO}$ & $58(30,2)$ & $33(28,2)$ & $23(28,7)$ & & $20(22,0)$ & $21(39,6)$ & $9(17,0)$ & \\
\hline $\mathrm{RN}$ & $25(13,0)$ & $31(26,5)$ & $23(28,7)$ & & $20(22,0)$ & $12(22,7)$ & $19(35,9)$ & \\
\hline
\end{tabular}

* Nem todos os estudantes responderam a todas as questôes.

GO: Goiás; RN: Rio Grande do Norte; SC: Santa Catarina; SP: São Paulo.

10,9 vezes maior do que pelos meios de comunicação e 14,1 vezes maior do que por meio de médicos $(\mathrm{p}<0,0001)$. Tem-se ainda que a chance de um estudante de Goiás adquirir conhecimento da anticoncepção de emergência pela escola é aproximadamente duas vezes maior que a chance de estudantes das outras regiões adquirirem informações por intermédio desta fonte $(\mathrm{p}=$ 0,0005) - não mostrado em Tabela/Figura.
Dos 581 alunos que responderam à questão sobre anticoncepção de emergência ser ou não abortiva, 207 (35,6\%) a consideravam um método que induz aborto. Não houve diferença estatisticamente significante dessa variável quando feita a comparação entre as regiões $(p=0,0503)$.

Cerca de $81 \%(n=473)$ dos adolescentes achavam que o uso de anticoncepção de emergência poderia trazer risco à saúde. Não se verifi- 
cou diferença dessa opinião entre as regiões $(\mathrm{p}=$ 0,5151). Em todas as regiões, os riscos mais citados pelos adolescentes foram malformação fetal em caso de uso de anticoncepção de emergência na vigência de gravidez e sangramento genital em grande quantidade.

\section{Experiência com anticoncepção de emergência}

Das 182 meninas que disseram conhecer anticoncepção de emergência, já tiveram relação sexual e responderam à questão sobre o uso do método, 76 (41,8\%) referiram já ter feito uso de anticoncepção de emergência; e dos 143 meninos, $32(22,4 \%)$ relataram que a parceira tinha usado, sendo que não se observou diferença estatística significante entre as regiões. Em São Paulo e Santa Catarina, o principal motivo do uso da anticoncepção de emergência foi acidente com preservativo; em Goiás e Rio Grande do Norte, ausência do uso de outros métodos contraceptivos.

A média e o desvio padrão do número de vezes que adolescentes do sexo feminino usaram anticoncepção de emergência foi $1,83 \pm 1,29$, variando de uma a dez vezes, sem diferença estatística entre as regiões.

Em todas as regiões, os amigos ou o farmacêutico/balconista da farmácia foram as principais fontes de indicação da anticoncepção de emergência para a adolescente. Apenas 5,3\% ( $\mathrm{n}=4$ ) das meninas adquiriram anticoncepção de emergência por meio de prescrição médica.

\section{Opinião em relação à anticoncepção de emergência}

Grande parte dos estudantes, 68,9\% ( $n=403$ ), acreditava que havia risco importante dos adolescentes negligenciarem o uso de métodos contraceptivos de rotina caso soubessem da possibilidade de usar anticoncepção de emergência, sendo que não se observou diferença significativa entre as regiões em relação a esta questão ( $\mathrm{p}=0$,3355).

Quando os adolescentes que nunca utilizaram o método foram questionados se usariam ou aconselhariam suas parceiras a usarem, 47,1\% ( $\mathrm{n}=144$ ) das meninas referiram que usariam, $17 \%$ ( $n=52)$ não usariam e $35,9 \%(n=110)$ não sabem se usariam. Não se observou diferença estatística em relação a essa informação entre as regiões ( $\mathrm{p}=0,0527)$. Entre os meninos, $48,7 \%$ ( $n=95$ ) aconselhariam sua parceira a usar anticoncepção de emergência se fosse necessário, $34,4 \%$ ( $\mathrm{n}=67$ ) não aconselhariam o uso e 16,9\% ( $\mathrm{n}=33$ ) não sabem se aconselhariam; neste caso também não foi observada diferença estatística entre as regiões $(\mathrm{p}=0,9244)$. Dentre os principais motivos do não uso, do não aconselhamento e de não saber se usariam ou aconselhariam tem-se a falta de informação suficiente sobre o método, como os possíveis efeitos colaterais, complicações para a mulher e para o feto no caso de gravidez e a percepção sobre o método ser abortivo.

Não se observou relação entre conhecimento e concordância em usar o método se fosse necessário. A maioria das que usariam anticoncepção de emergência achava que o método traz riscos à saúde, que a possibilidade de uso pode estimular os adolescentes a terem relação sexual desprotegida; porém a maioria não o considerava abortivo.

\section{Discussão}

A população estudada não é representativa dos universitários do país.

A idade média de início da atividade sexual verificada neste trabalho é superior à encontrada na literatura nacional: $15-16$ anos para as meninas e 13,9-14,5 anos para os meninos 41 . Contreras \& Hakkert 42 afirmam que o grau de escolaridade gera impacto diretamente proporcional à idade em que ocorre a iniciação sexual, portanto, o fato de os jovens entrevistados estarem freqüentando o ensino superior poderia justificar o adiamento do início da vida sexual observado.

O método contraceptivo mais utilizado pelos estudantes entrevistados foi o preservativo masculino. Trata-se de uma forma de contracepção amplamente difundida, foco de inúmeras campanhas educacionais, pois é um dos poucos métodos que confere ao indivíduo dupla proteção (DST e gravidez).

Proporção significativa de adolescentes recorreu à tabelinha e ao coito interrompido para evitar gravidez. É bem possível que os adolescentes desconheçam as altas taxas de falhas desses métodos (25\% e $19 \%$, respectivamente) e/ou se considerem tão onipotentes a ponto de acharem que não estão vulneráveis à gravidez e/ou DST, quando optam por esses métodos comportamentais 43 .

Foi elevada a porcentagem de estudantes que referiu não utilizar métodos contraceptivos em todas as relações sexuais: cerca de $30 \%$ dos jovens que participaram do estudo, em algum momento, estavam correndo risco de gravidez não planejada e/ou aquisição de DST nestes intercursos desprotegidos.

Observou-se neste trabalho maior porcentagem de estudantes que "já ouviram falar" e apresentavam conhecimentos específicos sobre anti- 
concepção de emergência do que a encontrada na grande maioria dos estudos recentemente publicados $26,27,44$. A justificativa para essa mais alta prevalência de conhecimento deve-se, provavelmente, à característica da população estudada, composta apenas por universitários que freqüentavam instituições públicas federais do país.

É interessante notar que "ter ouvido falar" sobre anticoncepção de emergência não significa conhecer o método, como demonstram outros trabalhos 23,44,45. Nenhum estudante respondeu que 120 horas é o tempo máximo que a anticoncepção de emergência pode ser utilizada após o intercurso sexual desprotegido, como se preconiza atualmente 46,47. Menos da metade dos estudantes respondeu que o tempo máximo para o uso da anticoncepção de emergência é de 72 horas, e quase metade respondeu que poderia ser usado de 24 a 48 horas após o coito desprotegido, possivelmente pela designação popular que chama a contracepção de emergência de "pílula do dia seguinte", nome infeliz, que pode persuadir a adolescente a achar que só até o dia seguinte ao ato sexual desprotegido a medicação fará algum efeito.

Embora a grande maioria dos estudantes soubesse, pelo menos, uma situação na qual o método pode ser usado, apenas um quinto dos estudantes sabia precisamente todas as situações nas quais a anticoncepção de emergência estava indicada. Menos da metade dos estudantes conhecia a real efetividade da anticoncepção de emergência quando comparada aos anticoncepcionais orais hormonais. Na verdade, os alunos sabem da existência da anticoncepção de emergência, mas não estão familiarizados com o método, o que contribui para as percepções negativas e conseqüente pouca utilização, como também é observado em inúmeras publicações 23,25,27,44,45.

É preocupante constatar que a principal fonte de aquisição de conhecimento sobre anticoncepção de emergência, na maioria das regiões, seja a escola, pois questões relevantes sobre o método são desconhecidas, remetendo à idéia de que o tipo de informação que as escolas estejam oferecendo possa ser insuficiente. Observou-se que mesmo em Goiás, onde há diferença significativa entre a aquisição da informação obtida na escola quando comparada a outros métodos, e onde quase $80 \%$ dos adolescentes obtêm informação de anticoncepção de emergência na escola contra $60 \%$ a $70 \%$ nas outras regiões (diferença esta também com significância estatística), estes adolescentes não possuem mais conhecimento sobre anticoncepcionais de emergência do que os das outras regiões aqui analisadas.
Idealmente seria, também, que a aquisição de conhecimentos sobre saúde sexual ocorresse de forma mais expressiva por intermédio de médicos ou outros profissionais de saúde, pois o conteúdo da informação fornecida pela mídia ou por amigos pode ser duvidoso e incompleto. É provável que os médicos não estejam aconselhando seus pacientes sobre anticoncepcionais de emergência. Em trabalho realizado com residentes de pediatria e pediatras da prefeitura do Município de São Paulo, obteve-se que cerca de $70 \%$ dos médicos não aconselhavam seus pacientes adolescentes sobre anticoncepção de emergência 14 .

Quando os meninos foram questionados sobre o uso de anticoncepção de emergência pela parceira, observou-se discrepância entre as porcentagens relatadas por eles e pelas meninas, mostrando que o parceiro desconhece os métodos contraceptivos utilizados pela parceira ou a atitude tomada em caso de falha no método contraceptivo utilizado. Como a maioria dos métodos contraceptivos é para ser utilizado pela mulher, prescindindo a escolha da participação masculina, se vincula a idéia de que a reprodução biológica e seu controle sejam de responsabilidade apenas do sexo feminino.

Observou-se que apesar de lacunas importantes no conhecimento, as jovens utilizam anticoncepcionais de emergência. Embora a prevalência de uso tenha sido superior à encontrada na literatura, este trabalho não contemplou situações nas quais as adolescentes poderiam ter utilizado anticoncepção de emergência e não o fizeram, ou seja, não se sabe se o método foi subutilizado por esta população.

Em todas as regiões, porcentagem pequena de meninas adquiriu anticoncepção de emergência por meio de prescrição médica. Inúmeros trabalhos mostram que os médicos temem prescrever anticoncepção de emergência para adolescentes receando que esta atitude possa promover comportamento sexual irresponsável ou uso inadequado, além de estarem pouco familiarizados com o método 14,15.

Adolescentes das quatro regiões acreditavam que a possibilidade de utilizar anticoncepção de emergência pode fazer com que a jovem adquira comportamento sexual de risco, aumentando a promiscuidade e diminuindo o uso de métodos contraceptivos de rotina. Observou-se neste trabalho que a média de vezes que as meninas utilizaram anticoncepção de emergência ficou próxima de duas (78\% usaram até duas vezes), não parecendo, portanto, que as adolescentes utilizam a anticoncepção de emergência como substituto de outros métodos. 
Percentual elevado de adolescentes achava que o uso de anticoncepção de emergência pode trazer riscos à saúde, e embora pouco mais da metade dos estudantes não considerassem anticoncepção de emergência abortiva, uma grande parcela acreditava que se trata de método que induz aborto. A literatura é categórica em afirmar que a anticoncepção de emergência não traz riscos à saúde, não causa infertilidade, não provoca sangramento ou alterações significativas no padrão menstrual, não interrompem gravidez estabelecida e, se usada na vigência de gestação, não é teratogênica 43,48 .

Considerar a anticoncepção de emergência abortiva e desconhecer as especificidades do método foram os principais motivos encontrados neste trabalho para o não uso de anticoncepção de emergência pelos estudantes entrevistados, como também é relatado na literatura 23,25,49.

Melhorar o conhecimento dos jovens sobre a anticoncepção de emergência, fornecendo a eles informações sobre os mecanismos de ação para desmistificar o conceito de anticoncepção de emergência ser abortiva, indicações e forma de uso, mostrando aos potenciais usuários que o método é seguro e efetivo, bem como orientar os adolescentes para que eles possam identificar situações de risco de gravidez, incluindo, sempre, os jovens do sexo masculino nestas discussões, poderia otimizar a utilização do método e prevenir inúmeras gestações indesejadas e abortos ilegais no Brasil.

A escolha de um grupo constituído por jovens universitários foi fundamentada na justificativa da alta escolaridade deste grupo, sendo que com esta informação algumas inferências podem ser feitas. Os resultados do estudo não podem ser extrapolados para a população de adolescentes em geral, mas se pode inferir que se o conhecimento desses estudantes tão diferenciados não é satisfatório, o dos adolescentes da população como um todo deve ser ainda mais deficiente.

Embora se tenha observado diferença estatisticamente significativa em muitos aspectos do conhecimento entre as regiões, não se observou nenhuma variável que pudesse explicar de maneira lógica estas diferenças. É provável que as variáveis estudadas não foram suficientes para explicar as diferenças obtidas e, apesar de existirem, elas não impactaram na experiência e na opinião dos adolescentes sobre o método nas diferentes regiões, podendo levar a crer que programas nacionais, sem necessidade de regionalização, podem ser conduzidos para melhorar o conhecimento sobre anticoncepção de emergência desses jovens.

\section{Resumo}

O objetivo deste estudo foi avaliar as diferenças regionais de conhecimento, opinião e uso de anticoncepção de emergência entre universitários brasileiros. Questionário semi-estruturado abordando conhecimento, opinião, experiência com anticoncepção de emergência e comportamento sexual foi aplicado a adolescentes de universidades brasileiras. Para análise estatística utilizou-se o teste exato de Fisher e ANOVA. Diferenças foram significantes quando o valor de $p<0,05$. Cerca de $96 \%(n=588)$ dos estudantes já tinham ouvido falar sobre anticoncepção de emergência, $19 \%(n=111)$ conheciam as situações nas quais está indicada, com diferenças inter-regionais; $42 \%$ das meninas que tinham vida sexual ativa já tinham feito uso do método; 35\%
( $n=207)$ dos estudantes consideravam contracepção de emergência abortiva e $81 \%(n=473)$ achavam que traz riscos à saúde, sem diferença significativa entre as regiões nas questões relativas a uso e à opinião. As diferenças inter-regionais sobre conhecimento não impactaram no uso e na opinião dos adolescentes sobre anticoncepção de emergência. Programas nacionais devem ser conduzidos para melhorar o conhecimento sobre o método.

Anticoncepção Pós-coito; Comportamento do Adolescente; Medicina do Adolescente; Adolescente; Sexualidade 


\section{Colaboradores}

F. C. Silva colaborou na coleta, organização dos dados e redação do artigo. M. S. S. Vitalle revisou o artigo e participou da redação e discussão. H. S. Maranhão contribuiu na coleta dos dados na Universidade do Rio Grande do Norte e na discussão do artigo. M. H. A. Canuto colaborou na coleta dos dados na Universidade Federal de Goiás e na discussão do artigo. M. M. S. Pires participou na coleta dos dados na Universidade Federal de Santa Catarina e na discussão do artigo. M. Fisberg colaborou com a coleta de informações e ajudou na redação do artigo, principalmente na discussão.

\section{Referências}

1. Heilborn ML, Aquino EML, Knauth DR. Juventude, sexualidade e reprodução. Cad Saúde Pública 2006; 22:1362-3.

2. Ministério da Saúde. Pesquisa Nacional de Demografia e Saúde da criança e da mulher - PNDS 2006. Brasília: Ministério da Saúde; 2009.

3. Heilborn ML, Aquino EML, Bozon M, Knauth DR, organizadores. O aprendizado da sexualidade: reprodução e trajetórias sociais de jovens brasileiros. Rio de Janeiro: Editora Garamond; 2006.

4. Ministério da Saúde. Marco teórico e referencial: saúde sexual e saúde reprodutiva de adolescentes e jovens. Brasília: Ministério da Saúde; 2007.

5. World Health Organization. Adolescent pregnancy: issues in adolescent health and development. Geneva: World Health Organization; 2004. (WHO Discussion Paper on Adolescence).

6. Rede Interagencial de Informações para a Saúde. Indicadores e dados básicos - Brasil - 2008 (IDB 2008). Brasília: Rede Interagencial de Informações para a Saúde, Ministério da Saúde; 2008.

7. Chalem E, Mitsuhiro SS, Ferri CP, Barros MCM, Guinsburg R, Laranjeira R. Gravidez na adolescência: perfil sócio-demográfico e comportamental de uma população da periferia de São Paulo, Brasil. Cad Saúde Pública 2007; 23:177-86.

8. Vieira LM, Goldberg TBL, Saes SO, Dória AAB. Abortamento na adolescência: um estudo epidemiológico. Ciênc Saúde Coletiva 2007; 12:1201-8.

9. Ministério da Saúde. Aborto e saúde pública: 20 anos de pesquisas no Brasil. http://portal.sau de.gov.br/portal/arquivos/pdf/aborto_e_sau de_publica_vs_preliminar.pdf (acessado em 10/ Mar/2009).
10. World Health Organization. Adolescent pregnancy. Geneva: World Health Organization; 2008.

11. Menezes G, Aquino EML. Pesquisa sobre o aborto no Brasil: avanços e desafios para o campo da saúde coletiva. Cad Saúde Pública 2009; 25 Suppl 2:S193-204.

12. Adesse L, Monteiro MFG. Magnitude do aborto no Brasil: aspectos epidemiológicos e sócio-culturais. Revista de Saúde Sexual e Reprodutiva; 2007. http://www.ipas.org.br/arquivos/factsh_mag.pdf (acessado em 10/Mar/2009).

13. Kosunen E, Vikat A, Rimpelä M, Rimpelä A, Huhtala H. Questionnaire study of use of emergency contraception among teenagers. BMJ 1999; 319:91.

14. Barry RF, Vitalle MSS, Silva FC. Emergency contraception: what do pediatrics know about this subject? Minerva Pediatr 2010; 62:153-60.

15. Goyal M, Zhao H, Mollen C. Exploring emergency contraception knowledge, prescription practices, and barries to prescription for adolescents in the emergency department. Pediatrics 2009; 123: 765-70.

16. Glasier A, Baird D. The effects of self-administering emergency contraception. N Engl J Med 1998; 399:1-4.

17. Lo SS, Fan SY, Ho PC, Glasier AF. Effect of advanced provision of emergency contraception on women's contraceptive behaviour: a randomized controlled trial. Hum Reprod 2004; 19:2404-10.

18. Walker DM, Torres P, Gutierrez JP, Flemming K, Bertozzi SM. Emergency contraception use is correlated with increased condom use among adolescents: results from Mexico. J Adolesc Health 2004; 35:329-34. 
19. Task Force on Postovulatory Methods of Fertility Regulation. Radomised controlled trial of levonorgestrel versus the Yuzpe regimen of combined oral contraceptives for emergency contraception. Lancet 1998; 352:428-33.

20. Trussell J, Koenig J, Ellertson C, Stewart F. Preventing unintended pregnancy: the cost-effectiveness of three methods of emergency contraception. Am J Public Health 1997; 87:932-37.

21. World Health Organization. Emergency contraception: a guide for service delivery. Geneva: World Health Organization; 1998.

22. Ministério da Saúde. Assistência ao planejamento familiar. Brasília: Ministério da Saúde; 1996.

23. Sorhaindo A, Becker D, Fletcher H, Garcia SG. Emergency contraception among university students in Kingston, Jamaica: a survey of knowledge, attitudes, and practices. Contraception 2002; 66:261-8.

24. Moreau C, Bouyer J, Goulard H, Bajos N. The remaining barriers to the use emergency contraception: perception of pregnancy risk by women undergoing induced abortions. Contraception 2005; 71:202-7.

25. Díaz S, Hardy E, Alvarado G, Ezcurra E. Acceptability of emergency contraception in Brazil, Chile, and Mexico. 2 - Facilitating factors versus obstacles. Cad Saúde Pública 2003; 19:1729-37.

26. Foster DG, Harper CC, Bley JJ, Mikanda J, Induni M, Saviano EC, et al. Knowledge of emergency contraception among women aged 18 to 44 in California. Am J Obstet Gynecol 2004; 191:150-6.

27. Bozkurt N, Korucuoglu U, Aksakal FN, Biri A, Ciftçi B, Maral I, et al. Turkish adolescent's knowledge on and attitude toward emergency contraception. J Pediatr Adolesc Gynecol 2006; 19:391-5.

28. Corbett PO, Mitchell CP, Taylor JS, Kemppainen J. Emergency contraception: knowledge and perceptions in a university population. J Am Acad Nurse Pract 2006; 18:161-8.

29. Puri S, Bhatia V, Swami HM, Singh A, Sehgal A, Kaur AP. Awareness of emergency contraception among female college students in Chandigarh, India. Indian J Med Sci 2007; 61:338-46.

30. Figueiredo R, Andalaft Neto J. Uso de contracepção de emergência e camisinha entre adolescentes e jovens. Revista da SOGIA-BR 2005; 6:2.

31. Bastos MR, Borges ALV, Hoga LAK, Fernandes MP, Contin MV. Práticas contraceptivas entre jovens universitárias: o uso da anticoncepção de emergência. Texto \& Contexto Enferm 2008; 17:447-56.

32. Araújo MSP, Costa LOBF. Comportamento sexual e contracepção de emergência entre adolescentes de escolas públicas de Pernambuco, Brasil. Cad Saúde Pública 2009; 25:551-62.

33. Instituto Brasileiro de Geografia e Estatística. Síntese de indicadores sociais 2006. http://www.ib ge.gov.br/home/presidencia/noticias/noticia_im pressao.php?id_noticia $=774$ (acessado em 30/ Set/2008)
34. McDonald G, Amir L. Women's knowledge and attitudes about emergency contraception: a survey in a Melbourne women's health clinic. Aust N Z J Obstet Gynaecol 1999; 39:460-4.

35. Virjo I, Kirkkola AL, Isokoski M, Mattila K. Use and knowledge of hormonal emergency contraception. Adv Contracept 1999; 15:85-94.

36. Abbott J, Feldhaus KM, Houry D, Lowenstein SR. Emergency contraception: what do our patients know? Ann Emerg Med 2004; 43:376-81.

37. World Health Organization. The adolescent health and development (AHD) unit - investing in a health sector response to adolescents and young people. http://www.searo.who.int/en/Section13/Sec tion1245.htm (acessado em 20/Jan/2009).

38. Associação Brasileira de Empresas de Pesquisa. Critério de classificação econômica Brasil. http:// www.abep.org/codigosguias/ABEP_CCEB.pdf (acessado em 20/Nov/2005).

39. Agresti A. Categorical data analysis. New York: John Wiley and Sons; 1990.

40. Neter J, Kutner M, Wasserman W, Nachtsheim C. Applied linear statistical models. $4^{\text {th }}$ Ed. New York: McGraw Hill; 1996.

41. Castro MG, Abramovay M, Silva LB. Juventudes e sexualidade. http://unesdoc.unesco.org/ images/0013/001339/133977por.pdf (acessado em 23/Mar/2009).

42. Contreras JM, Hakkert R. La sexualidad y la formación de uniones. In: Guzmán JM, Contreras JM, Hakkert R, Falconier de Moyano M, editores. Diagnóstico sobre salud sexual y reproductiva de adolescentes en América Latina y Caribe. México DF: United Nations Population Fund; 2001. p. 57-104.

43. American Academy of Pediatrics. Committee on adolescence. Contraception and adolescents. Pediatrics 2007; 120:1135-48.

44. Addo VN, Tagoe-Darko ED. Knowledge, practices, and attitudes regarding emergency contraception among students at a university in Ghana. Int J Gynaecol Obstet 2009; 105:206-9.

45. Araújo MSP, Costa LOBF. Comportamento sexual e contracepção de emergência entre adolescentes de escolas públicas de Pernambuco, Brasil. Cad Saúde Pública 2009; 25:551-62.

46. Rodrigues I, Grou F, Joly J. Effectiveness of emergency contraceptive pills between 72 and 120 hours after unprotected sexual intercourse. Am J Obstet 2001; 184:531-37.

47. Ellertson C, Evans M, Ferden S, Leadbetter C, Spears A, Johnstone K, et al. Extending the time limit for starting the Yuzpe regimen of emergency contraception to 120 hours. Obstet Gynecol 2003; 101:1168-71.

48. Sanfilippo J, Downing D. Emergency contraception: when and how to use it. J Fam Pract 2008; 57 (2 Suppl):S25-36.

49. Díaz S, Hardy E, Alvarado G, Ezcurra E. Acceptability of emergency contraception in Brazil, Chile, and Mexico. 1 - Perceptions of emergency oral contraceptive. Cad Saúde Pública 2003; 19:1507-17.

Recebido em 01/Abr/2010

Versão final reapresentada em 12/Jul/2010

Aprovado em 26/Jul/2010 